IJBPAS, February, 2022, 11(2): 500-509

ISSN: 2277-4998

International Journal of Biology, Pharmacy

and Allied Seiences (IJBPAS)

'A Bridge Betueen Caboratory and QRender'

Www.iibpas.com

\title{
FORMULATION AND EVALUATION OF HERBAL AQUEOUS GEL FOR MITIGATION OF MOUTH ULCER
}

\author{
S SRAVANI", CH SAI CHARITHA AND RAMA RAO NADENDLA \\ Department of Pharmaceutics, Chalapathi Institute of Pharmaceutical Sciences, Chalapathi \\ Nagar, Lam, Guntur-522034 \\ *Corresponding Author: Setti Sravani: E Mail: sravani.sette@gmail.com \\ Received 14 ${ }^{\text {th }}$ March 2021; Revised $18^{\text {th }}$ April 2021; Accepted 29 ${ }^{\text {th }}$ May 2021; Available online $1^{\text {st }}$ Feb. 2022 \\ https://doi.org/10.31032/IJBPAS/2022/11.2.5859
}

\begin{abstract}
The aim of this study was to formulate and evaluate herbal mouth ulcers gel using Psidium guajava L (Guava) leaves powder at different concentrations. Carbopol 934, Propylene glycol used as a gel base to form create herbal gel. Formulations were stored at 8,25 and $40^{\circ} \mathrm{C}$ for four weeks to investigate their stability. Color, centrifugation, liquefaction, phase separation, $\mathrm{pH}$. Powdered Guava leaves had no contact with Polymer, according to infrared spectroscopy. The gel was clear and homogeneous, with a $\mathrm{pH}$ range of 6 to 7. Viscosity, Spreadability and Extrudability properties are within the range which demonstrates the rheological behavior of the formulations. Antimicrobial trials of formulations revealed high effectiveness against Candida albicans and Aspergillus niger. Powdered guava leaves have a major antioxidant effect, according to experimental evidence from in vitro studies. The developed herbal formulation was stable, safe, and effective when compared to synthetic formulations was confirmed after the stability studies.
\end{abstract}

Keywords: Guava leaves powder, Aqeous Gel, Mouth ulcers, Carbopol934, Candida albicans INTRODUCTION

Gels are usually semi-solid formulations with a liquid phase thickened with additional ingredients. Topical gel formulations are used for skin application, percutaneous drug delivery, and local action on some mucosal surfaces. Commercially available gels 
containing synthetic and semi-synthetic active agents have many drawbacks, including tooth staining, itching, and burning sensations, due to the presence of high levels of alcohol and organic compounds. A mouth ulcer is a crack or breach in the mucous membrane that lines the mouth's interior. It is normally yellow or white in colour and appears as a depression in the mouth, which is the mucous membrane [1]. Biting the inner layer of the jaw, food allergies, hard teeth cleaning, hormonal changes, vitamin deficiency, bacterial infection, and diseases are all common causes of mouth ulcers. The current study looks at the use of herbal powdered Guava Leaves in medicinal gel for the treatment of mouth ulcers [2]. Psidium guajava L. is a species of Psidium guava is a medicinal plant that belongs to the Myrtaceae family. $P$. guajava is a well-known native medicinal plant that is used in a variety of indigenous medical systems. It's widely available all over India [3]. Polysaccharides, vitamins, essential oils, minerals, enzymes, proteins, sesquiterpenoid alcohols, triterpenoid acids, alkaloids, glycosides, hormones, flavonoids, tannins, and saponins are among the phytochemicals contained in guava. Guava, or Psidium guajava Linn, is abundant in lutein, zeaxanthine, and lycopene, as well as antioxidants and vitamins. phenols, triterpenes, carotenoids, lectins, fibre, fatty acids, quercetin, anthraquinones, phlobatannins, and cardiac glycosides are all abundant in guava [4] Guavas are high in dietary fibers, vitamins A and $\mathrm{C}$, folic acid, and the minerals potassium, copper, and manganese, as well as other nutrients. A single common guava $(P$. guajava) fruit contains around four times the amount of vitamin $\mathrm{C}$ as an orange and has a generally broad, low-calorie profile of essential nutrients [5]. Recently, much attention has been paid to the use of biofriendly and eco-friendly plant-based products for the treatment and prevention of various diseases, and it has been documented that conventional medicine is used by the majority of the world's population. India has a wide range of plants with medicinal properties. Medicinal plants may be used to discover viable substitutes for synthetic drugs. The use of medicinal plant-based medicine is steadily gaining popularity around the world. Twenty-five bestselling pharmaceutical innovator agents are extracted from natural ingredients, accounting for nearly half of the world's total. Because of their promise and the issue of drug resistance in microorganisms, medicinal plants are increasingly being used as raw materials in the development of new 
drugs. Both developed and developing countries are seeing an increase in demand for medicinal plants. Herbal medicinal plant research is one of the most common fields of study around the world .Herbal formulations are now being researched more extensively for their ability to prevent and treat oral disease . Herbs have long been used to clean teeth and cure dental disease, as well as to treat a variety of oral diseases. Oral cancer, dental caries, and periodontal disorders are some of the most common oral health issues. There is a well-established connection between the activities of microbial species living in the oral cavity's microbiota and oral diseases. The big need for alternative treatment, products and prevention options for oral diseases that are safe, economical and effective comes from the rise in disease incidence particularly in developing countries, increased resistance by pathogenic bacteria to currently used chemotherapeutics and antibiotics opportunistic infections in immuno compromized individuals and financial that is economical considerations in developing countries. Furthermore, for a developing country like India, allopathic medicine is too costly and resource intensive and it has only limited success in the prevention and treatment of oral diseases and periodontal disease. As a result, plant extracts used in conventional medicine and alternative products are still seen as viable alternatives to prescription and organic drugs. The current study looks at the use of herbal guava leaves in a prescription gel to treat mouth ulcers [2].

\section{MATERIALS AND METHODS}

Psidium guajava plant materials were obtained from a nearby agricultural farmhouse (lam, Guntur district). Fresh plant leaves were washed with distilled water before shade drying. The plant was authenticated at Acharyanagarjuna University (ANU), Department of Botany. All other analytical-grade ingredients i'ecarbopo1934, Methylparaben, Propyl paraben, propylene glycol were purchased from Loba Chemicals in Mumbai. All the chemicals are high grade purchased are compatable with skin.

\section{Preparation of herbal gel}

Gel was prepared by the method reported in [1] with minor modifications. Carbopol 934 $(0.5,1,1.5,2,2.5,3 \% \mathrm{w} / \mathrm{w})$ was dispersed in distilled water with continuous stirring until uniform mixture was obtained. The $\mathrm{pH}$ was adjusted to 6-6.5 using triethanolamine (TEA). Methyl paraben $(0.03 \% \mathrm{w} / \mathrm{w})$ and propyl paraben $(0.01 \% \mathrm{w} / \mathrm{w})$ dissolved in distilled water using water bath, propylene glycol $(5 \% \mathrm{w} / \mathrm{w})$ was added after cooling to 
this mixture Psidium guajava powder in various concentrations was added. Above mixture was properly mixed to the Carbopol
934 gel with continuous stirring. The composition of different formulations has been discussed in below Table 1.

Table 1: Composition of different formulations

\begin{tabular}{|c|c|c|c|c|c|c|}
\hline Ingredients (\%w/w) & F1 & F2 & F3 & F4 & F5 & F6 \\
\hline Guava leaves extract & 0.5 & 1 & 1.5 & 2 & 2.5 & 3 \\
\hline Carbopol934 & 1 & 1 & 1 & 1 & 1 & 1 \\
\hline Methyl paraben & 0.03 & $\mathbf{0 . 0 3}$ & $\mathbf{0 . 0 3}$ & $\mathbf{0 . 0 3}$ & $\mathbf{0 . 0 3}$ & $\mathbf{0 . 0 3}$ \\
\hline Propyl paraben & $\mathbf{0 . 0 1}$ & $\mathbf{0 . 0 1}$ & $\mathbf{0 . 0 1}$ & $\mathbf{0 . 0 1}$ & $\mathbf{0 . 0 1}$ & $\mathbf{0 . 0 1}$ \\
\hline Propylene glycol & 5 & 5 & 5 & 5 & 5 & 5 \\
\hline Triethanolamine & Q.S & Q.S & Q.S & Q.S & Q.S & Q.S \\
\hline Distilled water & Q.S & Q.S & Q.S & Q.S & Q.S & Q.S \\
\hline
\end{tabular}

\section{Evaluation parameters:}

\section{Physical appearance:}

Physical characteristics such as colour, odour, and consistency were visually examined.

\section{Colour:}

Visual inspection was used to verify the colour of the formulations.

\section{Consistency:}

The purity of the formulations was determined by putting them on the skin.

\section{Odour:}

The scent of the formulations was checked by dissolving the gel in water and smelling it. Table 2 demonstrates the physical tests of gel formulations.

\section{Measurement of $\mathrm{pH}$ :}

A automated $\mathrm{pH}$ meter was used to calculate the $\mathrm{pH}$ of herbal gel formulations. One gramme of gel was distributed in ten milliliters of distilled water and set aside for two hours. The $\mathrm{pH}$ of the formulation was calculated three times, with the average values being recorded. Table 2 shows the $\mathrm{pH}$ of the gel formulation.

\section{Homogeneity:}

After the gels were set in the jar, all formed gel formulations were visually inspected for homogeneity. They were examined for the existence of aggregates and the appearance of any aggregates. Table 2 shows the homogeneity of the gel composition [6].

\section{Spreadability:}

Glass slides and wooden blocks were used to test Spreadability. Weights of about $20 \mathrm{gm}$ were placed in the pan, and the time it took for the upper slide to fully separate from the fixed slide was recorded. On this ground slide, an excess of the $2 \mathrm{gm}$ gel under investigation was put. The gel was then sandwiched between this slide and another glass slide with a fixed ground slide and a hook attached. For 5 minutes, a $1 \mathrm{~kg}$ weighted was put on top of the slides to 
provide a uniform gel film and eliminate air between the slides. The excess gel was scraped away from the edges. The top plate was then pulled with the aid of a string attached to the hook, and the time it took the top slide to cover a distance of $7.5 \mathrm{~cm}$ was recorded in seconds. Spreadability is demonstrated by a shorter or smaller interval. The gel's Spreadability was measured using the formula below [7].

$\mathbf{S}=\mathbf{M} \times \mathbf{L} / \mathbf{T}$

Where,

S = Spreadability,

$M=$ Weight in the pan which is tied to the upper slide,

\section{$L=$ Length moved by the glass slide}

$T=$ Time in second taken to separate the slide completely each other

Viscosity:

Brookfield viscometer was used to assess viscosity (DV-III programmable Rheometer). At $250^{\circ} \mathrm{C}$, the rheological activity of the gels was examined. The measurements were taken over a speed range of 10 to $100 \mathrm{rpm}$, with 30 seconds between each speed, and then in reverse order [6].

\section{Extrudability:}

The gel formulations were filled and sealed in regular capped collapsible aluminium tubes. The thumb press was used to assess extrudability [6].

\section{Clarity:}

Visual testing was used to assess the clarity of all three batches [6].

\section{Gel strength:}

Gel intensity was measured by the amount of time it took the weight to reach the gel in seconds. $5 \mathrm{gm}$ of each of the optimize batches was taken as a sample, and $3.5 \mathrm{gm}$ of weight was added on the gel's surface. The time it takes for the weight to penetrate 0.5 $\mathrm{cm}$ into the gel in seconds. Table 3 was then used to report the gel strength [6].

\section{Stability studies:}

Formulation (F1-F6) was divided into four samples each and kept in four different stability chambers at $8^{\circ} \mathrm{C}, 25^{\circ} \mathrm{C}, 35^{\circ} \mathrm{C}$ and $40^{\circ} \mathrm{C}+75 \% \mathrm{RH}$ (relative humidity) respectively. These were observed for color, homogenecity, $\mathrm{pH}$, Viscosity and phase separation for period of one month at different time interval [8].

\section{Bioadhesive Strength:}

Glass slides and wooden blocks were used to assess bioadhesive strength. The force required to remove the formulation from the cellophane membrane is determined by bioadhesive strength. A particular amount of prepared gel, 1 gm, was mounted on a glass slide wrapped in cellophane membrane. The movable glass slide was mounted on the fixed slide, allowing for intimate interaction. 
To ensure intimate contact between the formulation and the membrane, a two-minute contact time was given. The weight was applied to the pan that came with the apparatus before the slides came loose. The formula was used to measure the bioadhesive force, which was expressed as the detachment stress in dyne/cm2 [9].

Detachment stress $=\mathbf{m} \cdot \mathbf{g} / \mathbf{A}$

Where,

$\mathbf{m}=$ Weight required to detach two glass slides from each other (gm).

$\mathrm{g}=$ Acceleration due to gravity i.e. $980 \mathrm{~cm} / \mathrm{s} 2$.

$A=$ Area of membrane exposed (cm2).

Antimicrobial activity:

Cup-plate approach was used to test the antifungal efficacy of both optimized and blank formulations in contrast to marketed antifungal formulations. Candida albicans was used to conduct the antifungal activity test. The prepared nutrient was brought in and poured onto sterile petri plates, which were then set aside to dry and cool. Candida albicans culture was then distributed using a micron wire loop. Drilling holes $4 \mathrm{~mm}$ deep was achieved with a sterile cork borer with a diameter of $6 \mathrm{~mm}$. Then, in each hole, position $0.5 \mathrm{gm}$ of gel from each formulation. Plates were then incubated for 48 hours at $27^{\circ} \mathrm{C}$. The inhibition zone (diameter in $\mathrm{mm}$ ) was then calculated [10].

\section{In-vitro anti-oxidant activity:}

DPPH (1, 1-Diphenyl-2-picryl-hydrazyl) free radical scavenging activity of powdered guava leaves was measured by DPPH assay. This methodology was described by Blois in 1958 where in that bleaching rate of the stable free radical, DPPH. In its radical from $\mathrm{DPPH}$ absorbed at $517 \mathrm{~nm}$, but upon reduction by an antioxidant or a radical species its absorbance decreases. Procedure, powder was dissolved in to methanol containing solvent. $0.1 \mathrm{~mm}$ solution of DPPH in ethanol was prepared and $1 \mathrm{ml}$ of this solution was added to different concentration of test tube. Concentration up to 50 to 300 $\mu \mathrm{g} / \mathrm{ml}$. After $30 \mathrm{~min}$ later, absorbance was measured at $517 \mathrm{~nm}$. Shows lower absorbance of reactant mixture then it indicates higher free radical scavenging activity. The experiment was repeated for three times. IC50 value in the tested ingredient is concentration required to scavenge 50\% DPPH free radical scavenging activity was calculated by the following equation. Antioxidant study by DPPH assay was reported in Table 5 [11].

DPPH radical scavenging activity (\%) = [(A517 control-A517sample)/A517 control]*100.

Where,

A0 $=$ Absorbance of DPPH.

A1 = Absorbance of DPPH solution in presence of the test solution. 


\section{RESULTS AND DISCUSSION}

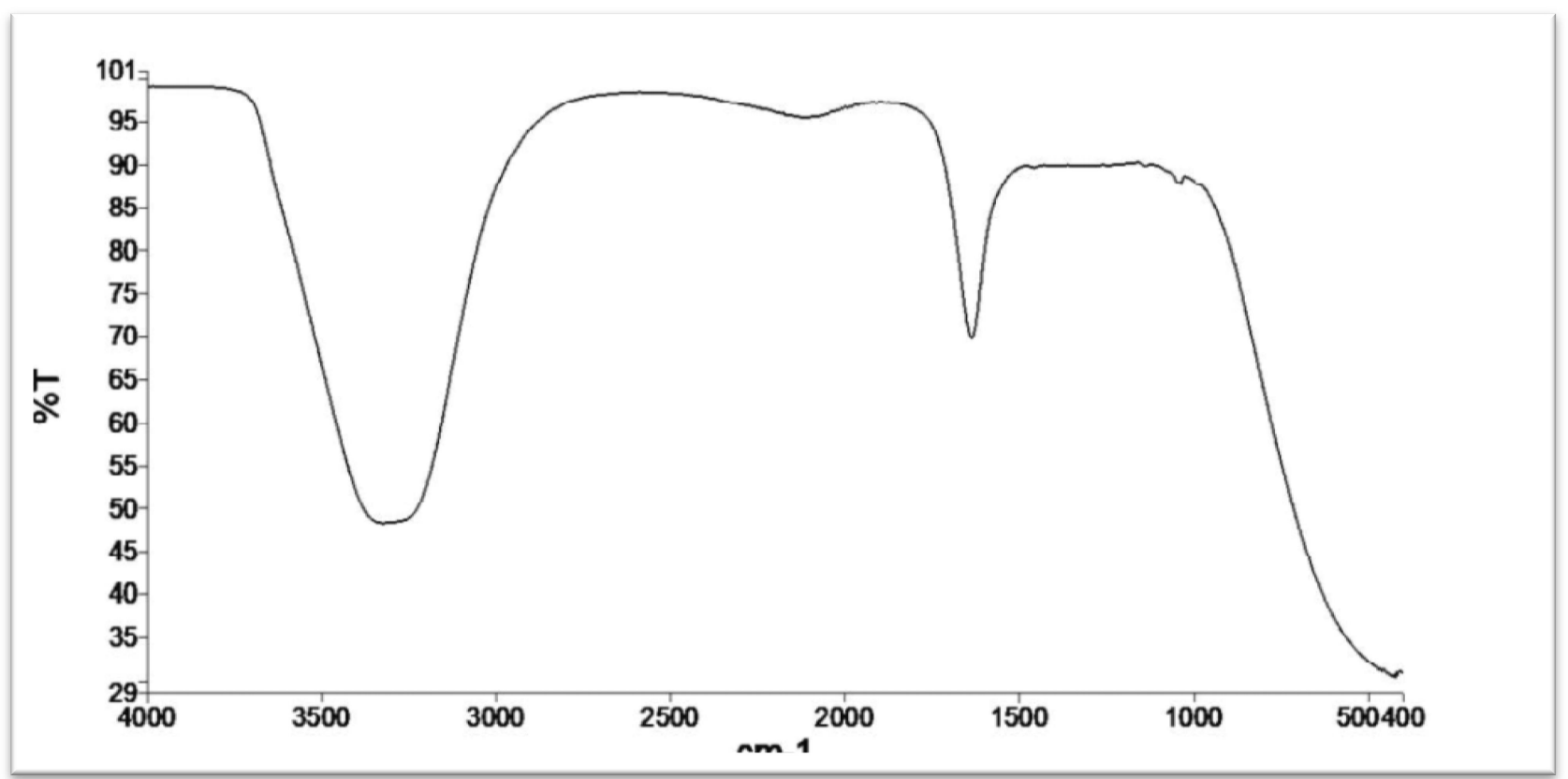

Figure1: IR spectra of powder guava leaves

Table 2: In vitro evaluation parameters

\begin{tabular}{|c|c|c|c|c|c|c|}
\hline Formulations & $\begin{array}{c}\text { Physical } \\
\text { Appearance }\end{array}$ & $\mathbf{p H}$ & Homogeneity & $\begin{array}{c}\text { Spreadability } \\
\text { (gm.cm/sec) }\end{array}$ & $\begin{array}{c}\text { Viscosity } \\
\text { (Pa.s) }\end{array}$ & Extrudability \\
\hline F1 & Greenish & $\mathbf{6 . 8}$ & Good & $\mathbf{5 . 1 0} \pm \mathbf{0 . 1}$ & $\mathbf{2 . 2 6 3} \pm \mathbf{0 . 0 0 4}$ & Good \\
\hline F2 & Greenish & $\mathbf{7 . 0}$ & Good & $\mathbf{5 . 2 6} \pm \mathbf{0 . 1}$ & $\mathbf{2 . 8 9 1} \pm \mathbf{0 . 0 0 1}$ & Good \\
\hline F3 & Greenish & $\mathbf{6 . 9}$ & Good & $\mathbf{5 . 0 5} \pm \mathbf{0 . 1}$ & $\mathbf{3 . 0 1 1} \pm \mathbf{0 . 0 1 0}$ & Good \\
\hline F4 & Greenish & $\mathbf{6 . 7}$ & Good & $\mathbf{5 . 5 8} \pm \mathbf{0 . 1}$ & $\mathbf{3 . 1 9 5} \pm \mathbf{0 . 0 2 0}$ & Good \\
\hline F5 & Greenish & $\mathbf{6 . 2}$ & Good & $\mathbf{5 . 6 9} \pm \mathbf{0 . 1}$ & $\mathbf{3 . 3 9 8} \pm \mathbf{0 . 0 0 1}$ & Good \\
\hline F6 & Greenish & $\mathbf{6 . 0}$ & Good & $\mathbf{6 . 0 1} \pm \mathbf{0 . 1}$ & $\mathbf{3 . 6 9 2} \pm \mathbf{0 . 0 3 1}$ & Good \\
\hline
\end{tabular}

Table 3: In vitro evaluation parameters

\begin{tabular}{|c|c|c|c|c|}
\hline Formulation & Bioadhesive strength & Gelling strength & \multicolumn{2}{c|}{ Stability study for 1 month } \\
\hline F1 & $\mathbf{2 6 4 8 . 0 8} \pm \mathbf{1 6 . 2 5}$ & $\mathbf{2 7} \pm \mathbf{0 . 5}$ & \multirow{3}{*}{ Open container } & \\
\\
\hline F2 & $\mathbf{2 9 0 3 . 4 1} \pm \mathbf{3 1 . 2 5}$ & $\mathbf{3 4} \pm 0.07$ & \multirow{3}{*}{ Not stable } & \\
\hline F3 & $\mathbf{3 2 1 4 . 3 6} \pm \mathbf{1 6 . 2 5}$ & $\mathbf{4 1} \pm \mathbf{0 . 2 5}$ & & \\
\hline F4 & $\mathbf{4 0 2 2 . 0 9} \pm \mathbf{2 2 . 2 5}$ & $\mathbf{4 2} \pm \mathbf{0 . 7 5}$ & \\
\hline F6 & $\mathbf{4 6 9 6 . 2 4} \pm \mathbf{3 9 . 0 5}$ & $\mathbf{4 9} \pm \mathbf{0 . 0 5}$ & \\
\hline
\end{tabular}

Table 4: In vitro Anti Fungal Study

\begin{tabular}{|c|c|c|}
\hline Formulation & \multicolumn{2}{|c|}{ Anti Fungal Study } \\
\hline Fungal Strength & Aspergillus niger (mm) & 17 \\
\hline Blank & 14 & $18 \pm 0.1$ \\
\hline F1 & $16 \pm 0.1$ & $20 \pm 0.4$ \\
\hline F2 & $19 \pm 0.1$ & $23 \pm 0.1$ \\
\hline F3 & $\mathbf{2 1} \pm 0.6$ & $25 \pm 0.6$ \\
\hline F4 & $\mathbf{2 5} \pm 0.1$ & $27 \pm 0.2$ \\
\hline F5 & $\mathbf{2 9} \pm 0.3$ & $28 \pm 0.3$ \\
\hline Marketed formulation & $33 \pm 0.6$ & $27 \pm 0.2$ \\
\hline
\end{tabular}




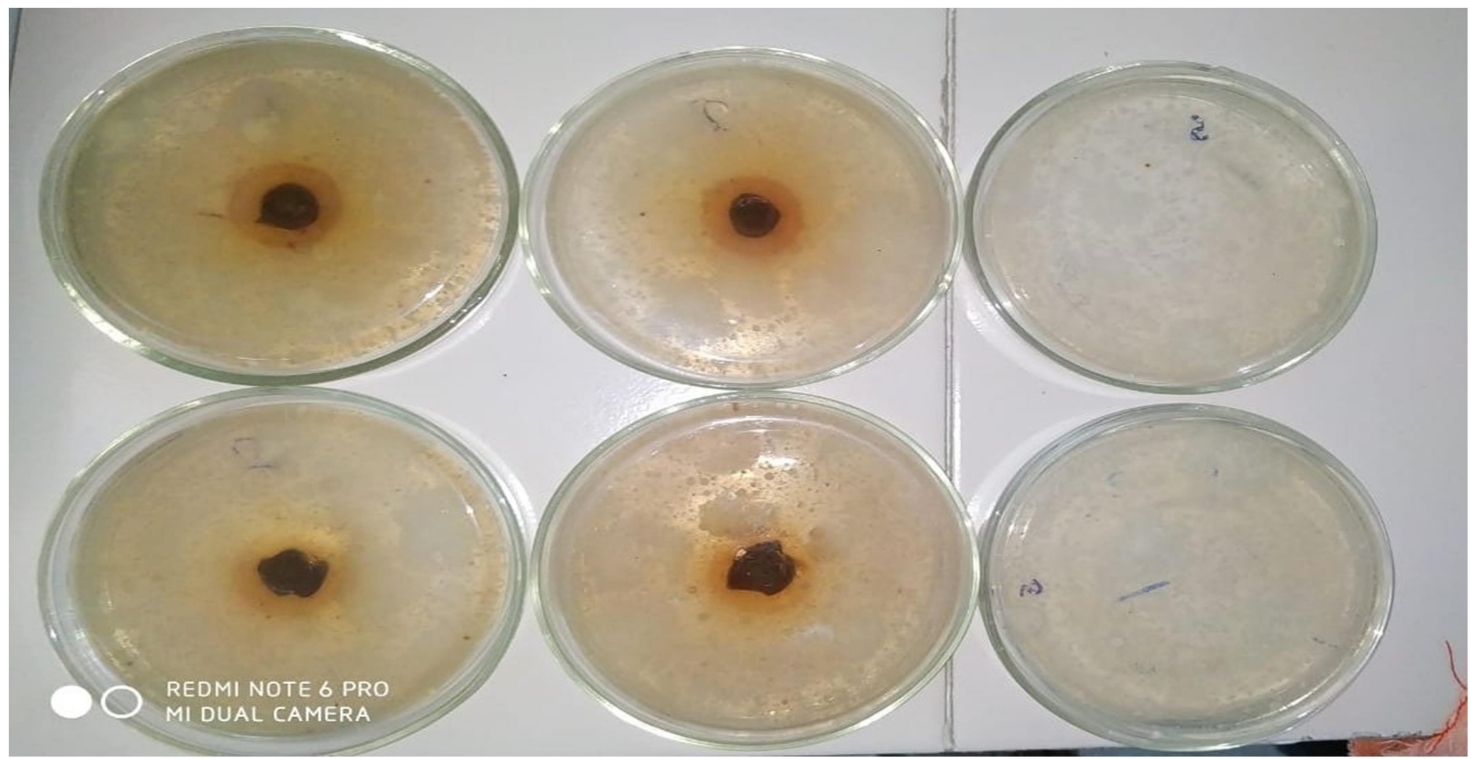

Figure 2: Anti Fungal activity of Guava gel

Table 5: In vitro Antioxident Study by DPPH Assay

\begin{tabular}{|c|c|c|c|}
\hline $\begin{array}{c}\text { Concentration } \mu \mathrm{g} / \mathrm{ml} \\
\text { (Powdered Guava leaves) }\end{array}$ & $\begin{array}{c}\text { \% Inhibition of powdered } \\
\text { guava leaves }\end{array}$ & $\begin{array}{c}\text { Concentration } \boldsymbol{\mu g} / \mathbf{m l} \\
\text { (Ascorbic acid) }\end{array}$ & $\begin{array}{c}\text { \% Inhibition of Ascorbic } \\
\text { acid }\end{array}$ \\
\hline 25 & $\mathbf{7 1 . 0 8 0} \pm \mathbf{0 . 0 4 5}$ & $\mathbf{2 5}$ & $\mathbf{8 9 . 7 7 0} \pm \mathbf{0 . 0 0 9}$ \\
\hline $\mathbf{5 0}$ & $\mathbf{7 5 . 3 4 2} \pm \mathbf{0 . 0 4 8}$ & $\mathbf{5 0}$ & $\mathbf{9 0 . 3 7 1} \pm \mathbf{0 . 0 1 2}$ \\
\hline 100 & $\mathbf{7 8 . 6 1 4} \pm \mathbf{0 . 0 8 5}$ & $\mathbf{1 0 0}$ & $\mathbf{9 2 . 4 2 3} \pm \mathbf{0 . 0 0 1}$ \\
\hline $\mathbf{1 5 0}$ & $\mathbf{8 0 . 9 7 4} \pm \mathbf{0 . 0 2 0}$ & $\mathbf{1 5 0}$ & $\mathbf{9 5 . 6 2 1} \pm \mathbf{0 . 0 1 5}$ \\
\hline 200 & $\mathbf{8 8 . 0 5 1} \pm \mathbf{0 . 0 1 0}$ & $\mathbf{2 0 0}$ & $\mathbf{9 9 . 5 4 9} \pm \mathbf{0 . 0 0 6}$ \\
\hline
\end{tabular}

The results clearly demonstrate that all of the gel formulations prepared have strong homogeneity and gelling properties. Both gel formulations had a $\mathrm{pH}$ that was consistent with the skin's usual $\mathrm{pH}$ range. Rheological activity was investigated using arheometer with a range of 2.262 to $3.392 \mathrm{~Pa}$.s. This implies that the gel was neither too thick nor too thin when it was made. Spreadability decreases as the viscosity of the solution increases, and vice versa, according to the research. The thumb was used to test extrudability, and it is easily extendable.
Both of the batches' gelling and bioadhesive strength were found to be within reasonable limits. A one-month stability analysis was conducted with open and closed containers, revealing that open container gel was not stable whereas close container gel was. When a prepared gel with an open container is exposed to room temperature, syneresis occurs, which means liquid exudates separate. Syneresis occurs when the interaction between particles in the dispersed process is so strong that they can no longer stand on their own. The gel shrinks as the 
dispersing medium is squeezed out in droplets. Syneresis is a form of instability found in aqueous gels. Only the elastic contraction of the polymer (polymeric molecules) allows the separation of a solvent process in a syneresis system (Allen L). All six batches of the established formulation showed antimicrobial activity against Aspergillus niger and Candida albicans, two of the most common microorganisms that cause mouth ulcers, and the formulation can also be used to treat mouth ulcer infection. The presence of flavonoids in powdered guava leaves was discovered in a DPPH assay sample, indicating that it had a strong antioxidant effect.

\section{CONCLUSION}

According to the findings of this study, the established herbal gel formulation is an important, therapeutically effective, and suitable vehicle for drug delivery at a low cost, but with a high potential. A newly formulated herbal gel formulation is ideal for the treatment of mouth ulcers.

\section{REFERENCE}

[1] Shaikh S, Shete A, Doijad R. Formulation and Evaluation Pharmaceutical aqueous gel of powdered Guava Leaves for Mouth Ulcer Treatment. 2018; 6(4): 32-8.

[2] Sing R, Bansal S, Mishra MK.
iMedPub Journals Formulation and Evaluation of Herbal Oral Gel Containing Extracts of Powdered Psidium guajava Linn Leaves with Curcuma longa Linn Rhizomes to Treat Mouth Ulcer. 2020; 1-7.

[3] Shruthi SD, Roshan A, Sharma S, Sunita S. Review article a review on the medicinal plant Psidium guajava LINN (Myrtaceae ). 2013; 3(2): 1628.

[4] Prashant P. Bhalchim, Manjusha N. Dole, Formulation and Evaluation of Herbal Gel Containing Psidium guajava Linn Leaves Extract, Asian Journal of Pharmaceutical Technology \& Innovation, 03 (15); 2015 .

[5] Prabhudesai AP, Station BR. Review Article Psidium guajava: Multipurpose Medicinal Herb. 2019; 59(21): 125-32.

[6] Kafle A, Mohapatra SS, Reddy I. A review on medicinal properties of Psidium guajava 2018; (August).

[7] Jadhav ST. Formulation and Evaluation Pharmaceutical Aqueous Gel of Powdered Guava, Aloe Vera and Acacia Leaves for Treatment of Mouth Ulcer. 2019; 8(5): 1693-7.

[8] Info A. F ormulation and evaluation 
of herbal antioxidant $\mathrm{N}$ ardostachys jatamansi collected from I ndian $\mathrm{H}$ imalayan face cream of region. 2014; 4(Suppl 2): 2-5.

[9] Shadab M, Shamsi S. Journal of Ayurveda and Integrative Medicine Design and Development of Unani Emulgel for Vitiligo. J Ayurveda Integr Med [Internet]. 2020; 11(3): 199-205. Available from: https://doi.org/10.1016/j.jaim.2018.01 .006

[10] Thirunavukkarasu P. Evaluation of Antioxidant Potential and Antibacterial Activity of Acalypha indica Linn. using in vitro model. 2011; (August 2014).

[11] Yadav SK, Mishra MK, Tiwari A, Shukla A. Emulgel: A New Approach For Enhanced Topical
Drug Delivery. 2017; 9(1).

[12] Das A, Ahmed AB. Natural permeation enhancer for transdermal drug delivery system and permeation evaluation: Natural Permeation Enhancer For Transdermal Drug Delivery System And Permeation Evaluation: A Review. 2017; (September). 